\title{
ARTICLE OPEN Design of high-strength refractory complex solid-solution alloys
}

Prashant Singh ${ }^{1}$, Aayush Sharma ${ }^{2}$, A. V. Smirnov ${ }^{1}$, Mouhamad S. Diallo², Pratik K. Ray ${ }^{1,3}$, Ganesh Balasubramanian ${ }^{4}$ and Duane D. Johnson (iD) 1,3

Nickel-based superalloys and near-equiatomic high-entropy alloys containing molybdenum are known for higher temperature strength and corrosion resistance. Yet, complex solid-solution alloys offer a huge design space to tune for optimal properties at slightly reduced entropy. For refractory Mo-W-Ta-Ti-Zr, we showcase KKR electronic structure methods via the coherent-potential approximation to identify alloys over five-dimensional design space with improved mechanical properties and necessary global (formation enthalpy) and local (short-range order) stability. Deformation is modeled with classical molecular dynamic simulations, validated from our first-principle data. We predict complex solid-solution alloys of improved stability with greatly enhanced modulus of elasticity ( $3 \times$ at $300 \mathrm{~K}$ ) over near-equiatomic cases, as validated experimentally, and with higher moduli above $500 \mathrm{~K}$ over commercial alloys ( $2.3 \times$ at $2000 \mathrm{~K}$ ). We also show that optimal complex solid-solution alloys are not described well by classical potentials due to critical electronic effects.

npj Computational Materials (2018)4:16; https://doi.org/10.1038/s41524-018-0072-0

\section{INTRODUCTION}

Nickel-based superalloys exhibit high-temperature strength, toughness, and oxidation resistance in harsh environments. ${ }^{1}$ Improving existing single-crystal alloys is unlikely as melting is near $1350^{\circ} \mathrm{C}$, and, heat treatment lowers this to $\sim 1270^{\circ} \mathrm{C}$. In highspeed turbines, melting reduces below $1250{ }^{\circ} \mathrm{C}$ at the zone between the bond coat (e.g., $\mathrm{NiAl}$ ) and the single-crystal blade. ${ }^{2} \mathrm{As}$ such, the engine efficiency and thrust-to-weight ratio can be improved by a guided search for new materials. High-entropy alloys based on refractory elements may achieve higher temperature operation with superior creep strength. ${ }^{3}$ Typical refractory high-entropy alloys exhibit a yield strength of 500-700 MPa at $1200^{\circ} \mathrm{C}$, surpassing Ni-based superalloys. ${ }^{4}$ Indeed, at elevated temperatures Mo-based alloys show good thermal (higher conductivities with lower strains ${ }^{5}$ ) and mechanical (machinability) ${ }^{6}$ properties, making them promising candidates.

Almost all high-entropy alloys for which mechanical properties have been reported are based on $\mathrm{Cr}$-Fe-Co-Ni with other elements added, e.g., $\mathrm{Al}^{7} \mathrm{Mn}^{8} \mathrm{Mo}^{9}$ and $\mathrm{Ti}^{10}{ }^{10} \mathrm{CoCrFeNi}$ exhibits very high compression strength at $300 \mathrm{~K}$, often exceeding $1500 \mathrm{MPa}$. Strains in as-cast condition do not often exceed $5-7 \%$, albeit a few exhibit $25-33 \% .{ }^{10,11}$ Annealing does improve ductility of as-cast alloys. ${ }^{11}$ As with conventional alloys, a rapid decrease in strength (i.e., Young's modulus, E) occurs above 0.6 of the melting temperature $T_{\mathrm{m}}$, and the strength of alloys approaches $100 \mathrm{MPa}$ at $1273 \mathrm{~K} .^{7}$

High-entropy alloys consist of $N(\geq 5)$ elements in nearequiatomic compositions $\left(c_{a} \sim 1 / N\right)$, giving maximal point (mixing) entropy $\left(S_{\mathrm{pt}}=-\sum_{a=1}^{N} c_{a} \ln c_{a} \stackrel{\max }{\longrightarrow} \ln N\right)$, that may better form solid solutions due to a compromise between the large $S_{p t}$ and a formation (mixing) energy $\Delta E_{\text {form }}$ that is not too positive (strongly clustering) nor too negative (strongly ordering). ${ }^{7}$ As for binary solid solutions, Hume-Rothery's rules ${ }^{12}$ for atomic size difference $(\delta)$, crystal structure, valence electron concentration (VEC), and electronegativity difference $(\Delta X)$ play a similar role in high-entropy alloy formation. The production of several single- or multiphase alloys with face-centered cubic (A1), body-centered cubic (BCC or A2), hexagonal close-packed (A3), or cubic diamond (A4) structures exhibiting enhanced high-temperature strength, ductility, fracture and creep resistance to corrosion, ${ }^{7-10,13,14}$ and thermal stability ${ }^{15}$ validates the concept of HEAs. ${ }^{7}$ MoWVNbTa, e.g., with a density of $12.2 \mathrm{~g} / \mathrm{cm}^{3}$, has a reported usable strength up to $1873 \mathrm{~K}^{4}$

Nonetheless, from an alloy design perspective, complex solidsolution alloys (CSAs) offer a huge design space to tune properties, especially considering the strong effects alloying has on electronic properties ("band" filling, hybridization, Fermi-surface nesting, ...), phase stability, and structure. The CSAs comprised of whole composition (Gibbs) space, however, high-entropy alloys are a subset of it. Optimized CSAs offer a slightly reduced entropy with a single-phase region, or two-phase region for enhanced mechanical properties, existing in a desired operational temperature range. ${ }^{16-18}$

Here we narrow the design of high-strength, refractory (Mo-W)$\mathrm{Ta}-(\mathrm{Ti}-\mathrm{Zr}$ ) alloys via KKR electronic structure methods within density functional theory (DFT) using the coherent-potential approximation (CPA) to handle chemical disorder and thermodynamic averaging. ${ }^{16-19}$ The well-established KKR-CPA predicts structural properties [e.g., Young's $(E)$ or bulk modulus $(B)$ ], and phase stability $\left(\Delta E_{\text {form }}\right.$ vs. $\left.\left\{c_{a}\right\}\right)$, as well as short-range order (SRO) via thermodynamic linear response, ${ }^{18,20-23}$ a method, in particular, that revealed the origin for Hume-Rothery's size-effect rule. ${ }^{21,24}$ Notably, global stability $\left(\Delta E_{\text {form }}\right)$ and local instability (SRO) should be jointly assessed: while segregation is expected for $\Delta E_{\text {form }}>0$, SRO can be segregating from local compositional instabilities even

\footnotetext{
${ }^{1}$ Ames Laboratory, U.S. Department of Energy, lowa State University, Ames, IA 50011, USA; ${ }^{2}$ Mechanical Engineering, lowa State University, Ames, IA 50011, USA; ${ }^{3}$ Materials Science \& Engineering, lowa State University, Ames, IA 50011, USA and ${ }^{4}$ Mechanical Engineering \& Mechanics, Lehigh University, Bethlehem, PA 18015, USA Correspondence: Prashant Singh (prashant@ameslab.gov) or Duane D. Johnson (ddj@ameslab.gov, ddj@iastate.edu)
}

Received: 8 November 2017 Revised: 26 February 2018 Accepted: 7 March 2018

Published online: 28 March 2018 
if $\Delta E_{\text {form }}<0$. To predict mechanical behavior (e.g., $E$ ) vs. temperature $(T)$ rapidly, we performed extensive molecular dynamics (MD) simulations based on semi-empirical potentials, validated in part by first-principles results (and also highlighting limitations of such methods). The tuned and proposed refractory quinary alloys and their properties are placed in context to HumeRothery-type design targets and compared to experiments.

\section{RESULTS AND DISCUSSION}

\section{Hume-Rothery design targets}

High-entropy alloys contain elements with $c_{\alpha} \sim 35-12$ at.\% $(N=$ $3-8)$. Trial and error has led to alloys with simple crystal structures, and a few with extraordinary properties, ${ }^{25}$ e.g., formability using size disparate elements for confusion by design. ${ }^{26}$ For CSA design of phase stability and of electronic and mechanical behavior, targets for DFT-based KKR-CPA are limited by extending HumeRothery ${ }^{12}$ criteria:

1. Size: solute and host atomic radii (in elemental solid) must differ by $<15 \% .{ }^{12,21}$ For CSAs, with $\bar{r}=\sum_{i=1}^{N} c_{i} r_{i}$, size limit in terms of standard deviation is sensible: $0 \leq \delta \leq 6 \%$, with $\delta=$ $100 \% \times\left[\sum_{i=1}^{n} c_{i}\left(r_{i}^{2}-\bar{r}^{2}\right) / \bar{r}^{2}\right]^{1 / 2}$.

2. Lattice: similar crystal structures for solute and host.

3. VEC: large solubility when solute and host have the same VEC. A metal dissolves one of higher (lower) valency to a greater (lesser) extent.

4. $X^{\prime} \mathrm{s}$ : if $\Delta x$ is too great, metals tend to form intermetallic compounds, not solid solutions.

5. $\Delta E_{\text {form: }}$ for $-11 \lesssim \Delta E_{\text {form }} \lesssim 5 \mathrm{mRy}$ CSAs stabilized in usable $T \mathrm{~s}$.

A few comments are warranted. In \#1, the 6-6.8\% achieves $15 \%$ rule for CSAs with $\sim 50 \%$ confidence level, an inequality also found empirically. ${ }^{27,28}$ Extending \#3 via electronic density of states concepts, A2 forms for $4<\operatorname{VEC}<6$ as stability increases when bonding $d$-states fill, and is maximal when half-filled $(\operatorname{VEC} \approx 6)$; antibonding states fill with VEC $>6$ (above a pseudogap, see Results) and stability decreases. Indeed, A2 CSAs are observed when VEC is $5 \pm 1 .^{29}$ For $6.8 \leq \mathrm{VEC} \leq 8$ other phases compete, e.g., $\mathrm{Fe}-\mathrm{Cr}$ has $\mathrm{VEC}=7$ (like $\mathrm{Mn}$ ) and constituent's structure are both $\mathrm{A} 2$, yet the CSA is unstable to the $\sigma$-phase, as often appears. ${ }^{27}$ Again, from band filling, A1 becomes more stable for VEC $>8 .^{30}$ CSAs are indeed observed to form within these rules. ${ }^{31} \Delta E_{\text {form }}$ lower limit in \#5 is set by $-T_{\mathrm{a}} S_{\mathrm{pt}}$, where annealing temperature (needed for kinetics) is $T_{\mathrm{a}} \sim 0.55 T_{\mathrm{m}}(\sim 1000-1650 \mathrm{~K}$ for refractories); upper limit is set such that miscibility gap $T_{\mathrm{c}}^{\mathrm{MG}}<T_{\mathrm{a}}$ (where $158 \mathrm{~K} \sim$ $1 \mathrm{mRy}$ ). For $\delta>5 \%$, CSAs with $\Delta E_{\text {form }}>5 \mathrm{mRy}$ form complex phases, but tend to form metastable metallic glasses for $\Delta E_{\text {form }}$ $<-11 \mathrm{mRy} .{ }^{32}$ Considering binaries and supercells, these limits for CSA formation are supported. ${ }^{33}$

As we have shown, transition temperatures from a (CSA) to $\beta$ (ordered or segregated) phases are well estimated from calculated $\Delta E_{\text {form }}$ 's: ${ }^{34,35}$ For segregating CSAs $\left(\Delta E_{\text {form }}>0\right), T_{c}^{\mathrm{MG}} \approx \Delta E_{\text {form }} / S_{\mathrm{pt}}{ }^{34}$ and, for ordering CSAs with $\Delta E_{\text {form }}^{a \rightarrow \beta}=\Delta E_{\text {form }}^{a}-\Delta E_{\text {form }}^{\beta}>0$, the order-disorder transition is $T_{c}^{\text {od }} \approx \Delta E_{\text {form }}^{a \rightarrow \beta 35}$. Notably, \#4 also reveals if vibrations are important, as vibrational entropy in binaries correlates as $\Delta S_{\text {vib }}=-\Delta X / 3 \quad( \pm 0.06 \Delta \chi) .{ }^{36}$ Thus, we may quickly estimate $T_{\mathrm{c}}$ for $d$-band CSAs as $T_{\mathrm{c}}^{\alpha \rightarrow \beta} \approx T_{\mathrm{c}, \mathrm{pt}}^{\alpha \rightarrow \beta}$ $\left[1+\Delta S_{\mathrm{vib}}^{a \rightarrow \beta} / \Delta S_{\mathrm{c}, \mathrm{pt}}^{a \rightarrow \beta}\right]^{-1}$, which reproduces measured trends without phonon calculations. ${ }^{34}$ These estimates are within $5-10 \% .{ }^{34,35}$

As a predictive guide, we use KKR-CPA results to tune $\left(\Delta E_{\text {form }}, \delta\right.$, VEC, and $\Delta X)$ vs. $\left\{c_{a}\right\}$ to find (Mo-W)-Ta-(Ti-Zr) alloys in fivedimensional (5D) space with better stability and mechanical properties. Results identify the stability of competing phases, possible multiphase regions, electronic properties, and practical design limits. We use the above criteria to restrict search space for mechanical simulations.

Here, via the KKR-CPA, we search all CSAs without restrictions on $\left\{c_{a}\right\}$, or the need for large supercells, as $A 1, A 2$ (A3) have only 1 (2) atoms per cell. In this quinary, atomic size of $\operatorname{Zr}(1.60 \AA)$ is largest, followed by $\mathrm{Ti}, \mathrm{Ta}$, and $\mathrm{W}$, Mo $(1.46,1.43$, and 1.37, $1.36 \AA$ ), where bandwidths (inversely related to atomic size) and alloy hybridization determine the effect of size. ${ }^{21}$ For $X$ (or $\Delta X^{\prime} s$ ), reflecting solubility and vibrational entropy, ${ }^{34,36}$ (W, Mo) have largest $X(2.36,2.16)$, followed by $(\mathrm{Ti}, \mathrm{Ta}, \mathrm{Zr})$ with $(1.54,1.50,1.33)$. From $\Delta X$ (Mo, W) would have the largest solubility (mixing) range, while $\% Z r$ is smaller based on $\delta$. Larger $\% W$ increases " $E$ " for engineering needs, but increases weight, and $\% \mathrm{Zr}$ reduces $\mathrm{Ti}$ content while positively impacting flow stress. ${ }^{37}$

\section{Design and assessment}

First, we exemplify in Fig. 1, top panel, our accuracy for $\Delta E_{\text {form }}$ vs. $x$ in $\mathrm{Ta}_{1-x} \mathrm{~W}_{x}$, which agrees well with measured values (within $5 \%$ ), and ordering enthalpies are low $(<310 \mathrm{~K})$ compared to melting. Also, we show results for specific ordered cells, which are compared to and agree well with other reliable band-structure methods [e.g., VASP pseudo-potential ${ }^{38}$ and full-potential linearaugmented plane wave ${ }^{39}$ ]. For $\mathrm{Mo}_{x}(\mathrm{WTaTiZr})_{1-x}$ in Fig. 1 (bottom panel), we find that $A 2$ is favored over $A 1$ or $A 3$, and that increasing \%Mo (larger $x$ ) helps stabilize $A 2$. So, in this work, we focus on A2 phase of (Mo-W)-Ta-(Ti-Zr) alloy. For $x \gtrsim 0.4$ A4 phase competes with $\mathrm{A} 2$, and Frank-Kasper phases, like $\mathrm{C} 15-\mathrm{Mo}_{2}(\mathrm{Ti}-\mathrm{Zr})$, may be anticipated. Usually, elements from group IIA/IVA of the periodic table, e.g., $\mathrm{Al} / \mathrm{Si}$, are added to stabilize or change, e.g., oxidation resistance. However, we find that adding Al stabilizes A2 phase up to $20 \% \mathrm{Mo}$, and similar behavior of $\mathrm{Al}$ addition has been

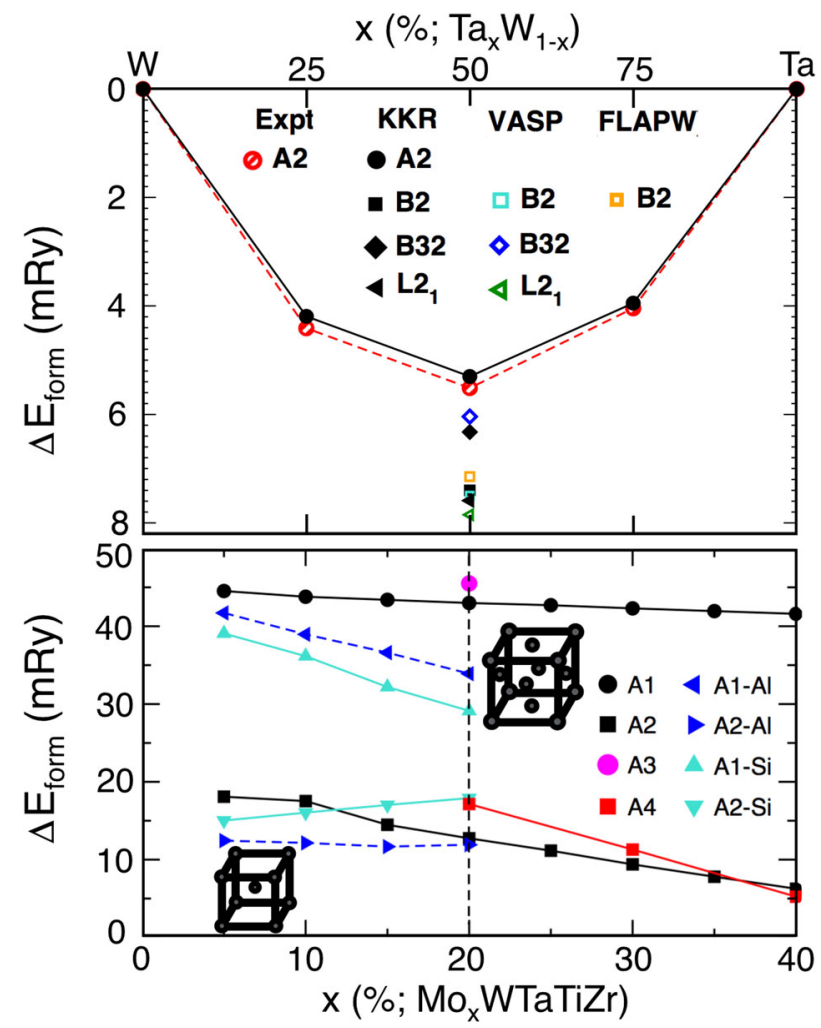

Fig. 1 (Top) Calculated formation energies $\left(\Delta E_{\text {form }}\right)$ for $\mathrm{Ta}_{x} \mathrm{~W}_{1-x}$ agree well with measured values. (Bottom) For $\mathrm{Mo}_{x}(\mathrm{WTaTiZr})_{1-x}$, $\left(\Delta E_{\text {form }}\right)$ vs. $x$ showing relative stability of $\mathrm{A} 1, \mathrm{~A} 2$, and $\mathrm{A} 3$ phases. While $\mathrm{A} 2$ is most favored, positive $\Delta E_{\text {form }}$ indicates segregation tendency, so $\Delta E_{\text {form }} \approx 0$ stabilizes the $\mathrm{A} 2$ phase 
seen in other alloys too. ${ }^{19}$ On the other hand, Si addition comes out to be energetically less favorable than Al addition.

High-throughput assessments. For (Mo-W)-Ta-(Ti-Zr) results are most easily presented in a cut through $5 \mathrm{D}\left\{c_{\alpha}\right\}$ space to visualize with only two parameters $(x, y)$ along lines or planes (Fig. 2), changing $\left\{c_{a}\right\}$ in obvious ways. For fast screening of (Mo-W)-Ta-(Ti$\mathrm{Zr}$ ) 5D composition design space, we used an estimate for lattice constant to perform "high-throughput" ${ }^{40-46}$ calculations to discover the best alloys in terms of phase stability and/or mechanical behavior. Specifically, we estimated alloy lattice constants via Vegard's rule, which is the concentration-weighted sum of volume-optimized elemental lattice constants in the parent alloys (A2) phase, or, simply, $a_{\text {alloy }}=\sum_{i}\left[c_{i}^{X} a_{i}^{X}\right]$, where $i=1,5$ and $X=$ $\mathrm{Mo}, \mathrm{W}, \mathrm{Ta}, \mathrm{Ti}, \mathrm{Zr}$. The estimated lattice constants are within $1-3 \%$ with respect to the optimized lattice constants for all considered compositions. To down-select regions of interest, we perform the calculation over the entire design space and chose increments in $\left\{c_{a}\right\}$ every $5 \%$ to sweep whole $5 \mathrm{D}$ space (Fig. 2). For selected alloys, we perform full lattice optimization to determine $\Delta E_{\text {form }}$ and $B$,

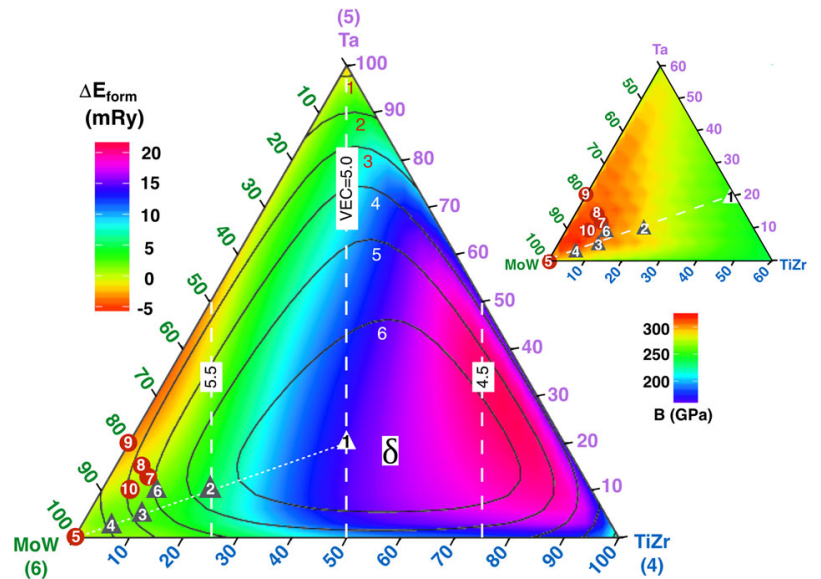

Fig. $2 \Delta E_{\text {form }}$ and $B$ (inset) vs. $(x, y)$ for $(\mathrm{MoW})_{x} \mathrm{Ta}_{y}(\mathrm{TiZr})_{1-x-y}$, a cut through $5 \mathrm{D}\left\{c_{\alpha}\right\}$ space containing equiatomic composition (C1), and plotted with vertical (dashed) lines of constant VEC (4-6) and contours of constant $\delta(1-6)$. Besides global stability $\left(\Delta E_{\text {form }}\right)$, alloys exhibit local instability (SRO) to segregation (triangles) or ordering (circles). DFT results are every $5 \%$ and interpolated. Maximal $B$ is near C7-10 with $\sim 300 \mathrm{GPa}$, where $\Delta E_{\text {form }} \sim 0$ and segregation is lost (Fig. 3) and detail the electronic structure (dispersion and density of states), and the thermodynamic SRO (incipient ordering) for alloy design.

Assessment and validation. We now assess CSAs that best satisfy design criteria, and local stability. Along with other targets, KKRCPA $\Delta E_{\text {form }}$ vs. $\left\{C_{a}\right\}$ for $(\mathrm{Mo}-\mathrm{W})_{x} \mathrm{Ta}_{y}(\mathrm{Ti}-\mathrm{Zr})_{1-x-y}$ are shown in Fig. 2. Clearly, $\Delta E_{\text {form }}$ for equiatomic case is too positive $(+12.7 \mathrm{mRy})$, and decomposition is expected (with $T_{c}^{\mathrm{MG}}=1244 \mathrm{~K}$ from estimates in Hume-Rothery section). Our calculated SRO also indicates phase decomposition at spinodal $T_{\mathrm{sp}}=1240 \mathrm{~K}$, agreeing with $T_{\mathrm{c}}^{\mathrm{MG}}$, in the near-equiatomic and Ti-Zr-rich alloys (Fig. 3). This predicted segregation is corroborated by our X-ray diffraction experiments (Fig. 4a) that indicate presence of two (major/minor) phases. The phases were indexed as a disordered A2 phase with $I m \overline{3} m$ space group, and a minor phase of $F d \overline{3} m$ space group. The $A 2$ lattice parameter was measured as $3.1713 \AA$ (standard deviation: $0.0002 \AA$ ). Figure $4 \mathrm{~b}$ shows the scanning electron microscope (SEM) micrograph of the alloy with a two-phase alloy evident. The major phase (A2) is Mo-, W-, and Ta-rich, with small amounts of Ti and $\mathrm{Zr}$ incorporated in it. Given the higher melting temperatures of Mo, W, and Ta, the major phase is likely to be the primary solidifying phase during the final step of casting. As this phase forms during casting, $\mathrm{Ti}$ and $\mathrm{Zr}$ are rejected into the surrounding liquid, which subsequently freezes. Hence, the minor phase is $\mathrm{Ti}$ and $\mathrm{Zr}$ rich and incorporates small amounts of the refractory metals.

To visualize key alloying effects for these CSAs, we plot in Fig. 5 the electronic dispersion and projected total density of states (TDOS), referenced to each alloy's Fermi energy, $E_{\mathrm{F}}$. With disorder, dispersion exhibits broadening in $E$ and $k$, showing that $k$ is a "good" (on the scale of the Brillouin zone (BZ)) but not an exact quantum number (as for zero-width, ordered bands); the width $\mathrm{d} \mathbf{k} \sim I_{\mathrm{e}}^{-1}\left(I_{\mathrm{e}}\right.$ is the electron-scattering length) and gives rise to increased residual resistivity, as may be calculated. ${ }^{47}$

Guided by such details, we can improve CSA properties. The equiatomic alloy has a TDOS with $E_{\mathrm{F}}$ not yet in the pseudogap between bonding and antibonding states (top, Fig. 5), so this alloy does not satisfy the design criteria. The VEC (the average electrons per atom outside the closed shells of the component atoms) is a dominant factor in controlling the phase stability of the alloys. The electronic states present on/near the $E_{\mathrm{F}}$ are chemically most active, which affect the chemical property of the alloy, i.e., more states at $E_{\mathrm{F}}$ destabilizes the alloy. This means, for such cases, adding or removing electron we can manipulate the electronic properties very quickly. By integrating states from $E_{\mathrm{F}}$ to the pseudogap for equiatomi case, 0.2 electrons are needed to fill
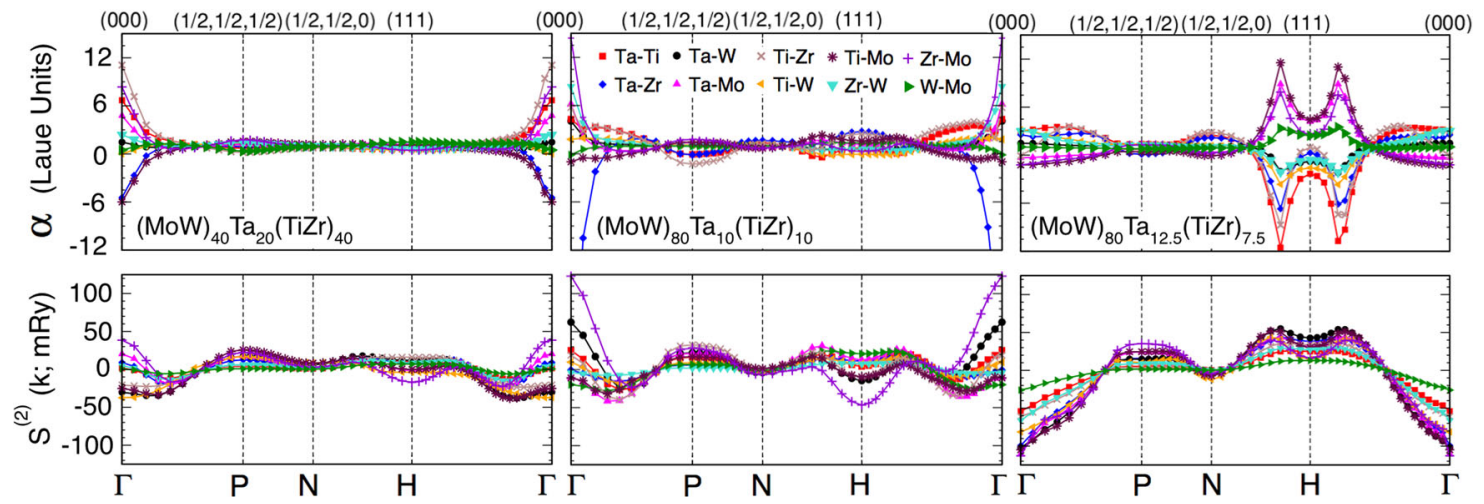

Fig. 3 For three alloys in Fig. 2, SRO correlations (top) $a_{\mu \nu}\left(\mathbf{k} ; 1.15 T_{\text {sp }}\right.$ ) and (bottom) energies $S_{\mu \nu}^{(2)}\left(\mathbf{k} ; 1.15 T_{\text {sp }}\right)$ plotted along high-symmetry lines in the Brillouin zone: (left) C1 $(x=2 / 5, y=x / 2)$ with $a_{\mathrm{TiZr}}(\Gamma)$ clustering with $T_{\mathrm{sp}}=1240 \mathrm{~K}$; (middle) $\mathrm{C} 6(x=4 / 5, y=1 / 10)$ with $a_{\mathrm{zrMo}}(\Gamma)$ clustering with $T_{\mathrm{sp}}=500 \mathrm{~K}$; and (right) $\mathrm{C7}(x=4 / 5, y=1 / 8)$ with $a_{\mathrm{TiMo}}\left(k_{0}\right)$ with incommensurate ordering $\left|\mathbf{k}_{0}\right|=0.7|\mathrm{~N}-\mathrm{H}|$ with $T_{\mathrm{sp}}=298 \mathrm{~K}$. For C1 [C6], Ti$\mathrm{Zr}[\mathrm{Zr}-\mathrm{Mo}]$ pairs dominate correlations, but clustering is driven by $S_{\mathrm{ZrMo}}^{(2)}(\Gamma)$. In C7, Ti-Mo dominate correlations, but Ti-Mo and Ta-W drive ordering 

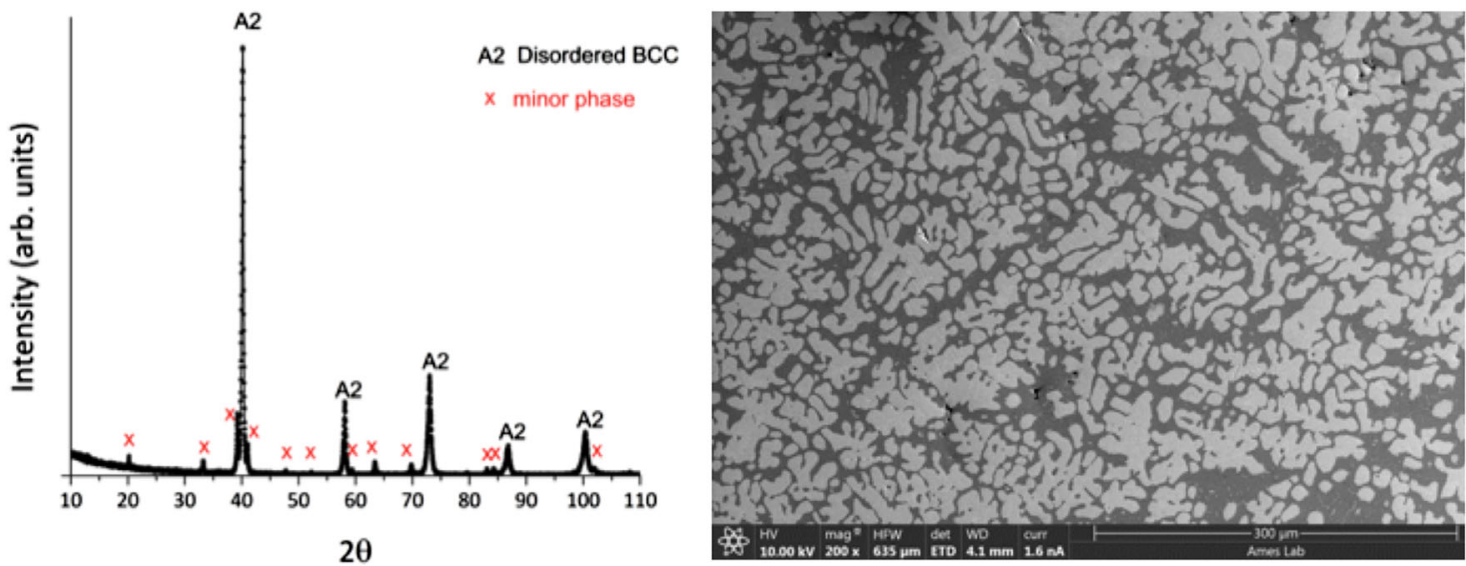

Fig. 4 a X-ray diffraction pattern from the equiatomic alloy; $\mathbf{b}$ backscattered SEM of the equiatomic alloy-the brighter contrast is the (Mo, W, Ta)-based solid solution, while the darker phase is the ( $\mathrm{Ti}, \mathrm{Zr})$-rich phase. The scale bar is $300 \mu \mathrm{m}$. Composition in major phase $(I \mathrm{~m}-3 \mathrm{~m})$ of $\mathrm{Mo} /$ $\mathrm{W} / \mathrm{Ta} / \mathrm{Ti} / \mathrm{Zr}$ is $22.96(1.79) / 33.63(2.57) / 28.31(0.44) / 11.19(1.79) / 3.90(0.52)$; and composition in minor phase $(\mathrm{Fd}(-3) \mathrm{m})$ of $\mathrm{Mo} / \mathrm{W} / \mathrm{Ta} / \mathrm{Ti} / \mathrm{Zr}$ is 8.44 $(0.82) / 0.89(0.15) / 3.06(0.22) / 32.61(0.35) / 55.00(0.87)$. Phase compositions as determined using EDS. Compositions are provided in at.\%. Standard deviations are provided in parentheses

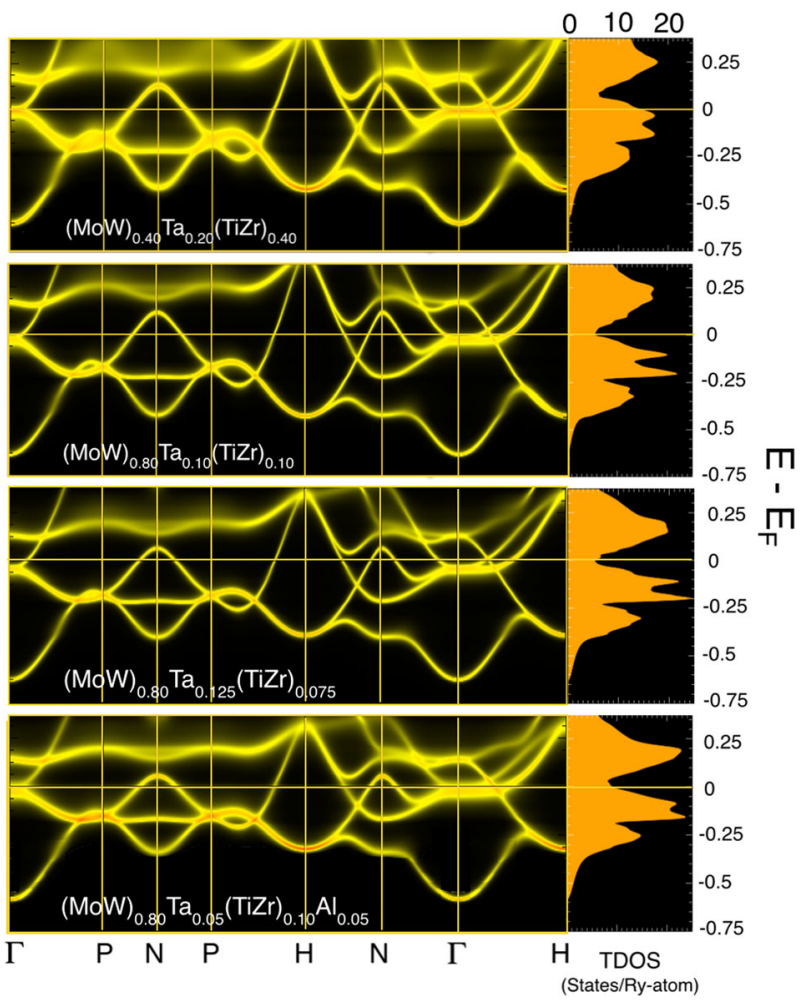

Fig. 5 For $\mathrm{C} 1, \mathrm{C} 6$, and $\mathrm{C} 7$ in Fig. 2, the electronic dispersion along Brillouin zone high-symmetry lines, and the projected total density of states (TDOS). Effects of Al doping is also shown (bottom). By tuning $\left\{c_{a}\right\}$ to add electrons, shift dispersion, or change disorder, the pseudogap is reached near $E_{\mathrm{F}}(0)$, better stabilizing the alloy and altering segregation

bonding states and improved stability. More at.\%Mo-W $(\mathrm{VEC}=6)$ adds electrons, moving $E_{\mathrm{F}}$ up (Fig. 5), and $\Delta E_{\text {form }}$ reduces to stabilize CSAs (Fig. 2). Adding small \%Ta helps in altering states near $E_{\mathrm{F}}$ : the flat bands near $\Gamma$ in Fig. 5 are moves from $E_{\mathrm{F}}$ for $C 7$, lowering $\Delta E_{\text {form, }}$ shown in Supplementary Figs. 2 and 3. We show in Fig. 3 that the SRO changes from clustering in C6 to ordering in C7, while $\Delta E_{\text {form }} \sim 0$. Here $\Delta E_{\text {form }}$ reduces quickly for MoW with a small \%Ta and \%TiZr, while bulk modulus $(B)$ increases quickly (inset Fig. 2). Notably, the dispersion of A2 metals is canonical when scaled by bandwidth (inverse atomic size), and so the behavior of the alloy dispersion is fairly generic and predominantly determined by relative composition and size, hybridization, and band filling.

To promote oxide-scale formation for protection, and lightweighting, $\mathrm{Al}$ is often added. In Fig. 5, 5\% Al added at the expense of Ta to C6 (whose $\Delta E_{\text {form }}=+0.70 \mathrm{mRy}$ ) increases disorder broadening (from Al $s p$ - $d$ hybridization) and causes $d$-state around $\Gamma$ (predominantly TiZr) to again straddle at $E_{\mathrm{F}}$. This Fermi-surface feature energetically destabilizes the alloy making $\Delta E_{\text {form }}$ much more positive $\left(+6.8 \mathrm{mRy}\right.$, with $\left.T_{\mathrm{c}}^{\mathrm{MG}}=828 \mathrm{~K}\right)$, which is also visible in SRO showing strong clustering behavior at $T_{\mathrm{sp}}=780 \mathrm{~K}$ (see Supplementary Fig. 4). The most significant Al-Mo pair suggest that Al will segregate to surfaces due its faster kinetics, as needed for oxide formation, i.e., adding $\mathrm{Al}$ at the expense of $\mathrm{Ta}$ or $\mathrm{TiZr}$ decreases VEC and drops $E_{\mathrm{F}}$ into localized $d$-states, reduces stability; so a balance must be struck by keeping some $\mathrm{Ta}$ and $\mathrm{TiZr}$ and making VEC high enough to be near $\Delta E_{\text {form }} \sim 0$ but with a large $B$ (Fig. 2). Unlike in other systems, Al is not generically a good A2 stabilizer, as it leads to larger electron scattering for reduced stability, increased resistivity, and decreased thermal transport, see ref. ${ }^{18}$ and references therein.

\section{Chemical SRO}

From KKR-CPA linear response (see Methods), we predict (Fig. 3) Warren-Cowley SRO (or atomic pair correlations) $a_{\mu v}(\mathbf{k} ; T)$, whose largest peak at wavevector $\mathbf{k}_{0}$ reveals the unstable (Fourier) modes to ordering, or clustering at $\Gamma=(000)$. As an alloying guide, SRO identifies pairs driving the instability, and predicts the spinodal $T_{\mathrm{sp}}$, where $a_{\mu \nu}^{-1}\left(\mathbf{k}_{0} ; T_{\mathrm{sp}}\right)=0$ signifying the absolute instability to this chemical fluctuation. ${ }^{18,19}$ In real space, pair probabilities are $P_{i j}^{\mu \nu}=c_{\mu}^{i} c_{\nu}^{j}\left(1-a_{\mu \nu}^{i j}\right)$, with $a_{\mu \nu}^{i \neq j}=0$ for no SRO, and $a<0 \quad(a>0)$ indicates ordering (clustering) with bounds of $\left[\min \left(c_{\mu}, c_{\nu}\right)\right]^{2}\left(c_{\mu} c_{\nu}\right)^{-1} \leq a_{\mu \nu}^{i \neq j} \leq 1$

Near-equiatomic alloys in Fig. 3 have maximal SRO peaks in $a_{\mu v}\left(\mathbf{k}_{0}\right.$ $=\Gamma ; T>T_{\mathrm{sp}}$ ) signaling spinodal (infinite wavelength) decomposition in specified pairs at $T_{\text {sp }}$ of $1240 \mathrm{~K}$ for $\mathrm{C} 1$, and at $500 \mathrm{~K}$ for C6. This $60 \%$ drop in $T_{\mathrm{sp}}$ is unsurprising given that $\Delta E_{\text {form }}$ reduces with Mo-W and Ta addition (Fig. 2). For C7, where $\Delta E_{\text {form }}$ has become slightly negative due to movement of bands present at $\Gamma$ away from $E_{\mathrm{F}}$ a weak incommensurate (long-period) ordering is found with SRO peak (Fig. 3) at $70 \%$ along $\mathrm{N}-\mathrm{H}$ at $\mathbf{k}_{0}=(0.85,0.15,0)$. This SRO arises from Fermi-surface nesting, ${ }^{48}$ with contributions at a radius of 
$\left|\mathbf{k}_{0}\right| \sim 0.86$, as confirmed along $\Gamma-H$ (Fig. 3). [SRO is B2 type if it peaks at $\mathbf{k}_{0}=H=\{100\}$, commensurate with A2 lattice.] For theory and detailed examples, see refs. ${ }^{18,19}$ We also show, in Supplementary Fig. 4 , that $5 \% \mathrm{Al}$ addition to the $\mathrm{C} 6$ alloy instigates a clustering instability. The Al-Mo pair drives spinodal decomposition at $T_{\mathrm{sp}}$ of $780 \mathrm{~K}$, which shows the tendency of $\mathrm{Al}$ to phase separate from $\mathrm{Mo}$, an indication that Al's clustering tendency might be helpful in promoting stable oxide layer at high temperatures. These results indicate that alloying may improve oxidation behavior, just as for $\mathrm{Fe}-\mathrm{Cr}$ with a narrow window for chromia formation. Clearly, KKR-CPA methods address profound electronic and alloying effects not possible from effective potentials, or methods that approximate disorder by ordered configurations.

\section{Deformation analysis}

Mechanical properties in CSAs have been studied at macro- and microscopic levels, ${ }^{49,50}$ but deformation analysis is key to establish high- $T$ structural candidates. We perform quasi-static uniaxial loading via MD simulations (see Methods) by deforming an ideal single-crystal alloy in small but finite steps and equilibrating after each step. For equiatomic case, $\langle 100\rangle$ compression (Fig. 6a) reveals a smooth stress-strain curve signaling simple plastic flow. In contrast, C3 (0.425 at.\%Mo) ideal crystal has stress drops and strain-hardening triggered by $<111>$ dislocations; a stress drop at 0.065 strain marks the initiation of dislocation with A2 Burgers vector, $\mathbf{b}=\frac{1}{2}<111>$, from 77 to $2000 \mathrm{~K}$.

Snapshots of the evolution show that dislocations (edge and screw type) triggered these instabilities (Fig. 6b). The defect mobility is affected by local distortions caused by the different sizes and modulus of the solutes. The rise and drop in stress with increasing strain in the ideal crystal corresponds to the defect evolution where new dislocations occur after every major stress drop followed by strain-hardening due to dislocation interactions and drag. An investigation of the local structural environment (Fig. $6 \mathrm{~b})$ reveals deviation from perfect $\mathrm{A} 2$, as yielding occurs for $300 \mathrm{~K}$. Shear bands (black) are promoted, denoting deformed regions with higher compression. At very high strain the interplay between edge and screw dislocations can be visualized via the band dynamics (Fig. 6b-4).

For engineering, Young's modulus $E=3(1-2 v) B$ is pertinent, so Poisson's ratio $v$ is also key. Small-deformation MD simulations determined $E$ and $v$ at $300 \mathrm{~K}$, and $E$ vs. $T$ was found from the elastic stress-strain curve (Fig. 6a). The KKR-CPA energy vs. a (A2 lattice constants) at $\left\{c_{a}\right\}$ determines the equilibrium $a_{0}, \Delta E_{\text {form }}$, and $B$ (Fig. $6 \mathrm{~d})$, all used in Fig. 2. We compare temperature-dependence of $E$, in Fig. 6c, calculated from MD, KKR-CPA (using Grüneisen approximation at low $T$ ), and experiments for commercial Morich TZM alloy, ${ }^{3}$ which again validate theory results. As MD is performed on ideal crystals, an "ideal" yield strength is obtained, with a qualitative relative change vs. temperature.

$E$ in equiatomic $C 1$, we performed indentation on samples prepared by arc-melting (see Methods), in Fig. 6c, and show that value from measurement $104 \pm 12 \mathrm{GPa}$ at $300 \mathrm{~K}$ compared very well to our predictions $115 \mathrm{GPa}$ from KKR-CPA and $120 \mathrm{GPa}$ from MD. As $B$ changes slowly for binary Mo-W (Fig. 6d), the Poisson effect (variation of $v$ ) controls strength, which requires W- or Mo-rich alloys for larger $E$ values (Fig. 6d). Similarly, for quinary $\left(\mathrm{Mo}_{1-z} \mathrm{~W}_{z}\right)_{0.85} \mathrm{Ta}_{0.10}(\mathrm{TiZr})_{0.05}$, we find that $\mathrm{C} 10 \quad(z=0.50)$ has strength similar to $C 4$ (Fig. $6 \mathrm{c}$ ). Whereas we predict a region around $\bar{C} \overline{10}(z=0.05$, highlighted in Fig. $6 \mathrm{~d}$, which is perpendicular to plane in Fig. 2 at (10) that shows both enhanced stability and $E$ (Fig. $6 \mathrm{c}$ ). For these CSAs, we find $3 \times$ larger $E$ than highentropy alloys at $300 \mathrm{~K}$, and alloys like $C \overline{1} 0$ have a much larger, less temperature-dependent modulus (Fig. 6c) above $500 \mathrm{~K}(2.3 \times$ at $2000 \mathrm{~K}$ ) over existing commercial TZM alloys, and lie midway between pure Mo and W, unlike TZM alloys.

Finally, one notable point, albeit not surprising, the classical MD simulations fail to represent properly the alloys that crossover from $\Delta E_{\text {form }}$ positive to negative (e.g., C4 to C7 or C3 to C10) in which electronic dispersion (not addressed by semi-empirical potentials) is controlling the materials physics. Hence, we plot only Cīo KKR-CPA values in Fig. $6 \mathrm{c}$, as MD values of $E$ vs. $T$ for $\mathrm{C} 10$ and
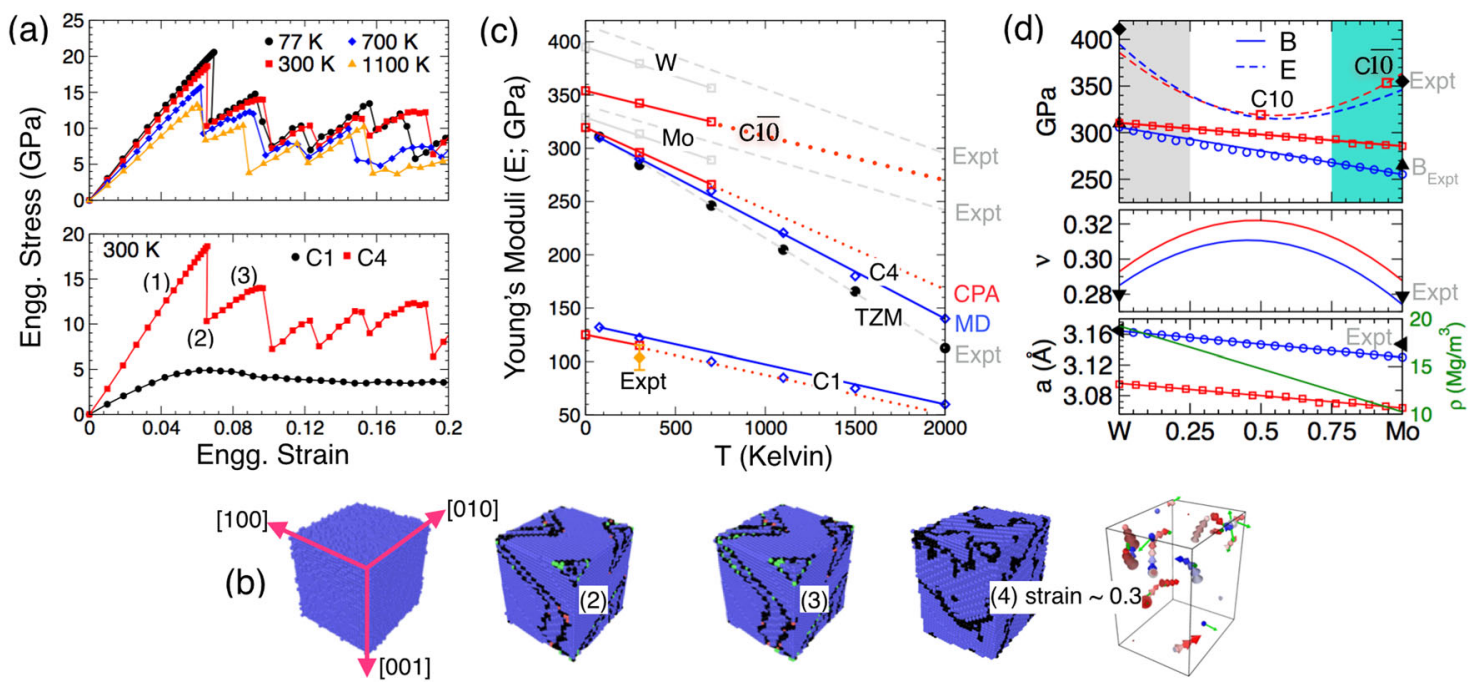

Fig. 6 a, bottom Simulated stress-strain for uniaxial quasi-static compression at $300 \mathrm{~K}$ on ideal crystals for equiatomic C1 alloy, which exhibits simple plastic flow, and for C4 alloy, where stress drops arise from dislocation motion and annihilation. a, top Stress-strain vs. $T$ from 77 to $1100 \mathrm{~K}$ for C4 alloy. b C4 strained elastically to 0.065 (b-1, blue), then, at yield, shear bands appear (b-2, black) accompanied by dislocations. At high strain rates ( 0.3), stronger edge (red) and screw (blue) components are found (b-4) with Burgers vector (green). c For (non)equiatomic alloys, $E$ vs. $T$ from classical MD [diamonds], KKR-CPA results [squares, extended to $600 \mathrm{~K}$ via Grüniesen theory, then extrapolated (dotted lines)], and measurements (Expt) for W, Mo, commercial TZM, ${ }^{3}$ and $\mathrm{C} 1$ alloy (present experiments). d For validation in $\mathrm{A}_{2} \mathrm{Mo}_{\mathrm{Z}} \mathrm{W}_{1-\mathrm{z}}$, measured (black symbols) and KKR-CPA results (blue lines and circles) for $B, E(\mathrm{GPa})$, lattice constant $a(\AA)$, and density $\rho\left(\mathrm{Mg} / \mathrm{m}^{3}\right)$, with Poisson ratio $(v)$ from MD. Also, predicted values are shown for quinary $\left(\mathrm{Mo}_{1-z} \mathrm{~W}_{z}\right)_{0.85} \mathrm{Ta}_{0.10}(\mathrm{TiZr})_{0.05}$ [red lines and squares], where $\mathrm{C} 10(z=0.5)$ response is similar to C4 in Fig. $6 c$. Design region: region around quinary $z=0.05$ (denoted $C \overline{1} 0$, at Mo-rich end of a line perpendicular to plane at $C 10$ in Fig. 2) shows enhanced $E$ (above Mo) and better T-dependent slope (Fig. 6c) from a favorable Poisson effect (green area in Fig. 6d). Above $500 \mathrm{~K}, \mathrm{C} \overline{1} 0$ has larger, less $T$-dependent $E$ than TZM $(2.3 \times$ at $2000 \mathrm{~K})$ 
$\mathrm{C} \overline{1} 0$ are similar to $\mathrm{C} 4$, whereas $\mathrm{C} \overline{1} 0$ values from first principles increases over C10, as expected from Fig. 6d.

From a design perspective, in general, alloys in CSAs have superior properties over near-equiatomic alloys (so-called highentropy alloys), although the design space becomes enormous. Using a first-principles KKR-CPA, we predicted the relative phase stability, dispersion, SRO (i.e., incipient long-range order, including $\left.T_{c}\right)$ and its electronic origin, and mechanical properties over all compositions as a design guide. Using electronic alloy design concepts and criteria, we identified higher-strength refractory (Mo-W)-Ta-(Ti-Zr) alloys from materials physics and engineering perspectives. Temperature-dependent deformation (most relevant the elastic behavior) in selected set of alloys was modeled using classical MD simulations, validated from first-principles data; we also identified failures in classical potentials that arose from dispersion effects.

Based on our calculation, we designed a Mo-rich region of improved stability with enhanced Young's moduli over highentropy alloys, as we confirmed experimentally, and an improved temperature-dependence above $500 \mathrm{~K}(2.3 \times$ at $2000 \mathrm{~K})$ over existing commercial alloys. Our electronic structure approaches and analysis of alloying and stability (formation energies, dispersion, and short-range ordering) highlight how instructive these details are in guiding design. The techniques are quite general for assessing any arbitrary CSAs, where alloying and nontrivial electronic effects play a key role.

\section{METHODS}

\section{DFT methods}

KKR electronic structure is used with the CPA to handle chemical disorder; ${ }^{16,17}$ screened CPA addresses Friedel screening from charge correlations. ${ }^{17}$ Scalar-relativistic effects are included (no spin orbit). Generalized gradient approximation to exchange correlation was included through use of $1 \mathrm{ibXC}$ libraries. ${ }^{51}$ CSAs require only one-atom (two-atom) cells for A1, A2 (A3). BZ integrations were performed with Monkhorst-Pack $k$-point method, ${ }^{52}$ with $12 \times 12 \times 12$ (6) for A1, A2 (A3) meshes. We used $300 k$-points in the irreducible $B Z$ to visualize dispersion along symmetry lines. Each scatterer's radii were defined by neutral "atoms in cell", with interstitial divided proportionally to each scatterer, to improve radial density representation near saddle points in the electronic density. ${ }^{53,54} \mathrm{We}$ chose $L_{\max }=3$ spherical-harmonic basis to include $s_{-}, p-, d$-, and $f$-orbital symmetries. Shallow core states were included in the valence in all calculations. A variational potential zero $v_{0}$ was used to yield kinetic energies nearing those of full-potential methods. ${ }^{55}$ For self-consistent densities, complex-energy contour integration ${ }^{56}$ used 20-point GaussLegendre semicircular contour.

\section{Chemical SRO}

From KKR-CPA linear response, we calculate SRO parameters, $a_{\mu \nu}(\mathbf{k} ; T)$, for $\mu$ $v$ pairs, ${ }^{18,19}$ as detailed elsewhere. ${ }^{20-23,48}$ Dominant pairs driving SRO are identified from pair-interchange energies, $S_{\mu \nu}^{(i, j)}(T)$, or curvature (concentration second variation) of the KKR-CPA grand potential, yielding energy cost for concomitant fluctuations of $c_{\mu}^{i}, c_{\nu}^{j}$ at atomic sites $i, j . S_{\mu \nu}^{(2)}(\mathbf{k} ; T)$ reveals the unstable (Fourier) modes with ordering wavevector $\mathbf{k}_{0}$ (or clustering at (000)), identifies the origin for phase transitions, and dictates the SRO: $a_{\mu \nu}^{-1}(\mathbf{k} ; T)=\left[c_{\mu}\left(\delta_{\mu \nu}-c_{\nu}\right)\right]^{-1}\left[\left(\delta_{\mu \nu} c_{\mu}^{-1}+c_{N}^{-1}\right)-\left(k_{\mathrm{B}} T\right)^{-1} S_{\mu \nu}^{(2)}(\mathbf{k} ; T)\right]$. The spinodal temperature, where $a_{\mu \nu}^{-1}\left(\mathbf{k}_{0} ; T_{\mathrm{sp}}\right)=0$, signifies an absolute instability to this fluctuation and provides an estimate for $T_{c}^{\mathrm{MG}}$ or $T_{\mathrm{c}}^{\text {od }} \cdot{ }^{18,19,57}$ For $N>2$, pairs driving ordering (clustering) will not necessarily be the same pairs that peak in the SRO due to the matrix inversion that relates them (Fig. 3).

\section{MD simulations}

Deformation is evaluated using Large-scale Atomistic/Molecular Massively Parallel Simulator package. ${ }^{58}$ The KKR-CPA structural parameters are used to validate potentials for finite- $T$ modeling. The force-field parameters for the quinary are established from available ternary EAM potentials. ${ }^{59}$ We verified similar hybrid potential parameter combinatorial technique for high-entropy alloy, like $\mathrm{Al}_{10} \mathrm{CrCoFeNi.}^{19}$ In $\mathrm{A} 2$ lattice ${ }^{60}$, with dimensions $(30 \times 30 \times 30)$ a $(54000$ atoms $)$, we distributed five elements via composition to form (Mo-W)-Ta-(Ti-Zr) solid solutions. Initially, the lattice was melted at $4000 \mathrm{~K}$ for $90 \mathrm{ps}$, followed by a quench to $300 \mathrm{~K}$ within $10 \mathrm{~ns}$. Uniaxial deformation was performed after equilibrating and relaxing the structure at high strain, as detailed in Supplementary Video $1 .{ }^{19}$

\section{Synthesis and characterization}

The equiatomic MoWTaTiZr was synthesized by arc-melting pellets of elemental powder blends (Alfa Aesar, purity $\geq 99.9 \%$ ) in an ultra-high-purity argon atmosphere on water-cooled copper hearth. Powders were used to reduce the large macro-segregation that occurs during casting when using elemental chips. With the significant difference in melting temperatures ( $3695 \mathrm{~K}$ for W vs. $1941 \mathrm{~K}$ for Ti) a three-step melting process was adopted. Step 1: W and Ta powders were mixed thoroughly in a SPEX 8000 mill, and pressed using a Carver hydraulic press; and the pellet was then arc-melted. Step 2: elemental blends of Mo, $\mathrm{Ti}$, and $\mathrm{Zr}$ were similarly mixed, pressed, and arc-melted. Step 3: both arc-melted buttons were re-melted together for a total of four times to ensure better homogeneity.

Phase analyses were carried out using a Philips PANalytical X-Ray Diffractometer, in a Bragg-Brentano geometry using $\mathrm{Cu}-\mathrm{Ka}$ radiation. Microstructure and phase compositions were analyzed using a FEI Helios NanoLab G3UC SEM, equipped with Oxford Energy Dispersive Spectroscopy system. Accelerating voltages of 10-15 kV were employed for imaging and compositional analyses. Compositions were measured at seven different locations for each phase, with the average composition and X-ray diffraction shown in Fig. 4. The diffraction pattern indicated the presence of two phases, indexed as a BCC (A2) phase with lattice parameter $3.1713(2) \AA$ and a minor phase with $F d \overline{3} m$ space group, like for B32 ( $\mathrm{NaTl}$ prototype) or $\mathrm{C} 15\left(\mathrm{MgCu}_{2}\right.$ prototype) structures, with lattice constant 7.6148(9) A.

\section{Nanoindentation}

Nanoindentation utilized a tribo-indenter HYSITRON TI-900 with a Berkovich $(3 \mu \mathrm{m})$ tip. The indenter control module applies a trapezoidal load on the sample for $10 \mathrm{~s}$, followed by $5 \mathrm{~s}$ rest, and unloads in $10 \mathrm{~s}$. To calibrate the sample measurements, which also determines the best applied load and optimum contact depth, the alloy was scanned (15 measurements) on an arbitrarily chosen sample location to optimize for force vs. displacement. For a minimum of $200 \mathrm{~nm}$ of contact depth, a $6000 \mu \mathrm{N}$ load was found to suffice. With these set, we indented the alloy at 30 manually chosen locations to measure the sample's elastic response; for the equiatomic case, the mean values were as follows: Young's modulus of $103.73 \pm 11.49 \mathrm{GPa}$; hardness of $4.6 \pm 0.34 \mathrm{GPa}$; and contact depth of $231.18 \pm 8.74 \mathrm{~nm}$.

\section{Data availability}

The authors declare that the data supporting the findings of this study are available within the paper and supplement. Also, the data that support the plots within this paper and other findings of this study are available from the corresponding author upon reasonable request.

\section{ACKNOWLEDGEMENTS}

Work supported by the U.S. Department of Energy (DOE), Office of Science, Basic Energy Sciences, Materials Science \& Engineering Division for theory/code development, and by the Office of Fossil Energy, Cross-cutting Research for application and validation for specific HEAs. Research was performed at lowa State University and Ames Laboratory, which is operated by ISU for the U.S. DOE under contract DE-AC0207CH11358. Work by A.S., M.S.D., and G.B. supported by the Office of Naval Research (grant N00014-16-1-2548), with computing resources from the Department of Defense High-Performance Computing Modernization Program.

\section{AUTHOR CONTRIBUTIONS}

P.S., A.S., G.B., and D.D.J. designed project. P.S. performed first-principles calculations. A.S. performed MD simulations. M.S.D. and P.K.R. performed experiments. P.S., A.S., and D.D.J. analyzed data and drafted manuscript. All authors contributed to the manuscript. 


\section{ADDITIONAL INFORMATION}

Supplementary information accompanies the paper on the npj Computational Materials website (https://doi.org/10.1038/s41524-018-0072-0).

Competing interests: The authors declare no competing interests.

Publisher's note: Springer Nature remains neutral with regard to jurisdictional claims in published maps and institutional affiliations.

\section{REFERENCES}

1. Pollock, T. M. \& Tin, S. Nickel-based superalloys for advanced turbine engines: Chemistry, microstructure, and properties. J. Propuls. Power 22, 361-374 (2006).

2. Balsone, S. J. et al. In Hemker, K. J. (ed) Proc. 3rd International Symposium on Structural Intermetallics, 99-108 (Jackson Hole, WY, USA, Sept. 23-27, 2001).

3. Shinno, H., Kitajima, M. \& Okada, M. Materials selection for reduced activation of fusion reactors. J. Nucl. Mater. 155-157, 290-294 (1988).

4. Senkov, O. N., Wilks, G. B., Scott, J. M. \& Miracle, D. B. Mechanical properties of $\mathrm{Nb}_{25} \mathrm{Mo}_{25} \mathrm{Ta}_{25} \mathrm{~W}_{25}$ and $\mathrm{V}_{20} \mathrm{Nb}_{20} \mathrm{Mo}_{20} \mathrm{Ta}_{20} \mathrm{~W}_{20}$ refractory high entropy alloys. Intermetallics 19, 698-706 (2011).

5. Shafeie, S. et al. High-entropy alloys as high-temperature thermoelectric materials. J. Appl. Phys. 118, 184905 (2015).

6. Perepezko, J. H. Materials science. The hotter the engine, the better. Science $\mathbf{3 2 6}$ 1068-1069 (2009).

7. Yeh, J.-W. et al. Nanostructured high-entropy alloys with multiple principal elements: novel alloy design concepts and outcomes. Adv. Eng. Mater. 6, 299-303 (2004).

8. Zhou, Y. J., Zhang, Y., Wang, Y. L. \& Chen, G. L. Microstructure and compressive properties of multicomponent $\mathrm{Al}_{x}\left(\mathrm{TiVCrMnFeCoNiCu}_{100-x}\right.$ high-entropy alloys. Mater. Sci. Eng. A 454, 260-265 (2007).

9. Kao, Y. F., Chen, T. J., Chen, S. K. \& Yeh, J.-W. Microstructure and mechanical property of as-cast, -homogenized, and -deformed $\mathrm{Al}_{x} \mathrm{CoCrFeNi}(0 \leq \mathrm{x} \leq 2)$ highentropy alloys. J. Alloy Compd. 488, 57-64 (2009).

10. Zhou, Y. J., Zhang, Y., Wang, Y. L. \& Chen, G. L. Solid solution alloys of AlCoCr$\mathrm{FeNiTi}_{x}$ with excellent room-temperature mechanical properties. Appl. Phys. Lett 90, 81904 (2007).

11. Zhang, K. B. et al. Annealing on the structure and properties evolution of the CoCrFeNiCuAl high-entropy alloy. J. Alloy Compd. 502, 295-299 (2010).

12. Hume-Rothery, W., Smallman, R. W. \& Haworth, C. W. The Structure of Metals and Alloys (The Institute of Metals, London, 1969).

13. Gludovatz, B. et al. A fracture-resistant high-entropy alloy for cryogenic applications. Science 345, 1153-1158 (2014).

14. Li, Z., Pradeep, K. G., Deng, Y., Raabe, D. \& Tasan, C. C. Metastable high-entropy dual-phase alloys overcome the strength-ductility trade-off. Nature 534, 227-230 (2016).

15. Dolique, V., Thomann, A. L., Brault, P., Tessier, Y. \& Gillon, P. Thermal stability of AlCoCrCuFeNi high entropy alloy thin films studied by in-situ XRD analysis. Surf Coat. Technol. 204, 1989-1992 (2010).

16. Johnson, D. D., Nicholson, D. M., Pinski, F. J., Gyorffy, B. L. \& Stocks, G. M. Densityfunctional theory for random alloys: total energy within the coherent-potential approximation. Phys. Rev. Lett. 56, 2088-2091 (1986).

17. Johnson, D. D. \& Pinski, F. J. Inclusion of charge correlations in calculations of the energetics and electronic structure for random substitutional alloys. Phys. Rev. $B$ 48, 11553-11560 (1993).

18. Singh, P., Smirnov, A. V. \& Johnson, D. D. Atomic short-range order and incipient long-range order in high-entropy alloys. Phys. Rev. B 91, 224204 (2015)

19. Sharma, A., Singh, P., Liaw, P. K., Johnson, D. D. \& Balasubramanian, G. Atomistic clustering-ordering and high-strain deformation of an $\mathrm{Al}_{0.1} \mathrm{CrCoFeNi}$ highentropy alloy. Sci. Rep. 6, 31028 (2016).

20. Staunton, J. B., Johnson, D. D. \& Pinski, F. J. Theory of compositional and magnetic correlations in alloys: Interpretation of a diffuse neutron-scattering experiment on an iron-vanadium single crystal. Phys. Rev. Lett. 65, 1259-1262 (1990).

21. Pinski, F. J. et al. Origins of compositional order in NiPt alloys. Phys. Rev. Lett. 66 766-769 (1991)

22. Staunton, J. B., Johnson, D. D. \& Pinski, F. J. Compositional short-range ordering in metallic alloys: band-filling, charge-transfer, and size effects from a first-principles all-electron Landau-type theory. Phys. Rev. B 50, 1450-1472 (1994).

23. Althoff, J. D., Johnson, D. D., Pinski, F. J. \& Staunton, J. B. Electronic origins of ordering in multicomponent metallic alloys: application to the $\mathrm{Cu}-\mathrm{Ni}$-Zn system. Phys. Rev. B 53, 10610-10625 (1996).

24. Maddox, J. News \& Views: making sense of binary alloys. Nature 349, 649 (1991)

25. Huang, P. K., Yeh, J.-W., Shun, T. T. \& Chen, S. K. Multi-principal-element alloys with improved oxidation and wear resistance for thermal spray coating. Adv. Eng. Mater. 6, 74-78 (2004).
26. Greer, A. L. Confusion by design. Nature 366, 303-304 (1993)

27. Tsai, M.-H. \& Yeh, J.-W. High-entropy alloys: a critical review. Mater. Res. Lett. 2, 107-123 (2014).

28. Yang, X. \& Zhang, Y. Prediction of high-entropy stabilized solid-solution in multicomponent alloys. Mater. Chem. Phys. 132, 233-238 (2012).

29. Mizutani, U. Hume-Rothery Rules for Structurally Complex Alloy Phases (CRC Press, New York, USA, 2010).

30. Skriver, H. L. The LMTO Method. (Springer-Verlag, Berlin, 1984).

31. Zhang, Y. et al. Microstructures and properties of high-entropy alloys. Progr. Mater. Sci. 61, 1-93 (2014).

32. Inoue, A., Zhang, T. \& Masumoto, T. Reductilization of embrittled LaAINi amorphous alloys by viscous flow deformation in a supercooled liquid region. J. NonCryst. Solids 156-158, 473-480 (1993).

33. Troparevsky, M. C., Morris, J. R., Kent, P. R. C., Lupini, A. R. \& Stocks, G. M. Criteria for predicting the formation of single-phase high-entropy alloys. Phys. Rev. X 5 011041 (2015).

34. Zarkevich, N. A., Tan, T. L. \& Johnson, D. D. First-principles prediction of phasesegregating alloy phase diagrams and a rapid design estimate of their transition temperatures. Phys. Rev. B 75, 104203 (2007).

35. Alam, A., Kraczek, B. \& Johnson, D. D. Structural, magnetic, and defect properties of Co-Pt-type magnetic-storage alloys: density-functional theory study of thermal processing effects. Phys. Rev. B 82, 024435 (2010).

36. Delaire, O., Swan-Wood, T. \& Fultz, B. Negative entropy of mixing for vanadiumplatinum solutions. Phys. Rev. Lett. 93, 185704 (2004).

37. Lu, J., Ravichandran, G. \& Johnson, W. L. Deformation behavior of the $\mathrm{Zr}_{41.2} \mathrm{Ti}_{13.8} \mathrm{Cu}_{12.5} \mathrm{Ni}_{10} \mathrm{Be}_{22.5}$ bulk metallic glass over a wide range of strain-rates and temperatures. Acta Mater. 51, 3429-3443 (2003).

38. Kresse, G. \& Hafner, J. Ab initio molecular dynamics for liquid metals. Phys. Rev. $B$ 47, 558-561 (1993).

39. Gulans, A. et al. exciting: a full-potential all-electron package implementing density-functional theory and many-body perturbation theory. J. Phys. Condens. Matter 26, 363202 (2014)

40. Curtarolo, S. et al. The high-throughput highway to computational materials design. Nat. Mater. 12, 191-201 (2013).

41. Curtarolo, S., Morgan, D., Persson, K., Rodgers, J. \& Ceder, G. Predicting crystal structures with data mining of quantum calculations. Phys. Rev. Lett. 91, 135503 (2003).

42. Setyawan, W. \& Curtarolo, S. High-throughput electronic band structure calculations: challenges and tools. Comput. Mater. Sci. 49, 299-312 (2010).

43. Jain, A. et al. A high-throughput infrastructure for density functional theory calculations. Comput. Mater. Sci. 50, 2295-2310 (2011).

44. Wang, S., Wang, Z., Setyawan, W., Mingo, N. \& Curtarolo, S. Assessing the thermoelectric properties of sintered compounds via high-throughput ab-initio calculations. Phys. Rev. X 1, 021012 (2011).

45. Koinuma, H. \& Takeuchi, I. Combinatorial solid-state chemistry of inorganic materials. Nat. Mater. 3, 429-438 (2004).

46. Spivack, J. L. et al. Combinatorial discovery of metal co-catalysts for the carbonylation of phenol. Appl. Catal. A 254, 5-25 (2003).

47. Butler, W. H. Theory of electronic transport in random alloys: Korringa-KohnRostoker coherent-potential approximation. Phys. Rev. B 31, 3260-3277 (1985).

48. Althoff, J. D., Johnson, D. D. \& Pinski, F. J. Commensurate and incommensurate ordering tendencies in the ternary fcc Cu-Ni-Zn system. Phys. Rev. Lett. 74, 138-141 (1995).

49. Giwa, A. M., Liaw, P. K., Dahmen, K. A. \& Greer, J. R. Microstructure and small-scale size effects in plasticity of individual phases of $\mathrm{Al}_{0.7} \mathrm{CoCrFeNi}$ high entropy alloy. Extrem. Mech. Lett. 8, 220-228 (2016).

50. Zou, Y., Maiti, S., Steurer, W. \& Spolenak, R. Size-dependent plasticity in an $\mathrm{Nb}_{25} \mathrm{Mo}_{25} \mathrm{Ta}_{25} \mathrm{~W}_{25}$ refractory high-entropy alloy. Acta Mater. 65, 85-97 (2014).

51. Marques, M. A. L., Oliveira, M. J. T. \& Burnus, T. Libxc: a library of exchange and correlation functionals for density functional theory. Comput. Phys. Commun. 183 2272-2281 (2012).

52. Monkhorst, H. J. \& Pack, J. D. Special points for Brillouin-zone integrations. Phys. Rev. B 13, 5188-5192 (1976).

53. Alam, A. \& Johnson, D. D. Optimal site-centered electronic structure basis set from a displaced-center expansion: Improved results via a priori estimates of saddle points in the density. Phys. Rev. B 80, 125123 (2009).

54. Johnson, D. D., Smirnov, A. V. \& Khan. S. N. MECCA: Multiple-scattering Electronic structure Calculations for Complex Alloys (KKR-CPA Program, ver. 2.0) (lowa State University and Ames Laboratory, 2015).

55. Alam, A. \& Johnson, D. D. Structural properties and relative stability of (meta) stable ordered, partially ordered, and disordered Al-Li alloy phases. Phys. Rev. B 85, 144202 (2012).

56. Johnson, D. D., Pinski, F. J. \& Stocks, G. M. Self-consistent electronic structure of disordered $\mathrm{Fe}_{0.65} \mathrm{Ni}_{0.35}$. J. Appl. Phys. 57, 3018-3020 (1985). 
57. Singh, P., Smirnov, A. V. \& Johnson, D. D. Ta-Nb-Mo-W refractory high-entropy alloys: anomalous ordering behavior and its intriguing electronic origin. Preprint at https://arxiv.org/abs/1711.10591 (2017).

58. Plimpton, S. Fast parallel algorithms for short-range molecular dynamics. J. Comput. Phys. 117, 1-19 (1995).

59. Zhou, X. W., Johnson, R. A. \& Wadley, H. N. G. Misfit-energy-increasing dislocations in vapor-deposited CoFe/NiFe multilayers. Phys. Rev. B 69, 144113 (2004).

60. Featherston, F. H. The Elastic Constants of Tantalum, Tungsten and Molybdenum. Thesis, Naval Postgraduate School (1963).

Open Access This article is licensed under a Creative Commons Attribution 4.0 International License, which permits use, sharing, adaptation, distribution and reproduction in any medium or format, as long as you give appropriate credit to the original author(s) and the source, provide a link to the Creative Commons license, and indicate if changes were made. The images or other third party material in this article are included in the article's Creative Commons license, unless indicated otherwise in a credit line to the material. If material is not included in the article's Creative Commons license and your intended use is not permitted by statutory regulation or exceeds the permitted use, you will need to obtain permission directly from the copyright holder. To view a copy of this license, visit http://creativecommons. org/licenses/by/4.0/.

(c) The Author(s) 2018 